\title{
STATEMENT
}

\section{Seminar Report From the 2014 Taiwan Society of Inflammatory Bowel Disease (TSIBD) Spring Forum (May 24th, 2014): Crohn's Disease Versus Intestinal Tuberculosis Infection}

\author{
Meng-Tzu Weng ${ }^{1,2}$, Shu-Chen Wei ${ }^{2}$, Chun-Che Lin ${ }^{3}$, Yuk-Min Tsang ${ }^{4}$, Chia-Tung Shun ${ }^{5}$, Jann-Yuan Wang ${ }^{2}$, \\ Ming-Jium Shieh², Cheng-Yi Wang' ${ }^{2}$ Jau-Min Wong' ${ }^{2}$ \\ Department of Internal Medicine, Far-Eastern Memorial Hospital, New Taipeil, Department of Internal Medicine, National Taiwan University \\ Hospital and College of Medicine, Taipei ${ }^{2}$, Department of Internal Medicine, Chung Shan Medical University Hospital, Taichung, Department \\ of Radiology, Far-Eastern Memorial Hospital, New Taipei ${ }^{4}$, Pathology and Forensic Medicine, National Taiwan University Hospital and College \\ of Medicine, Taipei ${ }^{5}$, Taiwan
}

Since Taiwan is an endemic area for tuberculosis (TB), differential diagnosis between the intestinal TB and Crohn's disease is an important issue. The steering committee of Taiwan Society of Inflammatory Bowel Disease (TSIBD) has arranged a seminar accordingly on May 24th, 2014 and the different point of views by gastroenterologist, radiologist, pathologist and infectious disease specialist were suggested to help the proper diagnosis and management of these two diseases. (Intest Res 2015;13:6-10)

Key Words: Crohn disease; Tuberculosis, intestine

\section{INTRODUCTION}

The diagnosis of IBD has to be made after excluding the possibility of infection. Since Taiwan is an endemic area for tuberculosis (TB), it is always required to be differentiated the TB from CD as always. To this issue, the steering committee of Taiwan Society of IBD (TSIBD) has arranged a seminar accordingly on May 24th, 2014 and a report of it has been generated afterwards. From the different point of views by gastroenterologist, radiologist, pathologist and infectious disease specialist, we hope this report can help our follow

Received January 5, 2015. Revised January 6, 2015.

Accepted January 6, 2015.

Correspondence to Jau-Min Wong, Department of Internal Medicine, National Taiwan University Hospital, National Taiwan University College of Medicine, No.7 Chung-Shan South Road, Taipei 101, Taiwan. Tel: +886-2-

23123456, Fax: +886-2-23947927,E-mail: jmwong@ntu.edu.tw

Financial support: None. Conflict of interest: None. clinicians to understand more about the diagnosis and management of these two diseases.

\section{EPIDEMIOLOGY}

The incidence of TB in Taiwan was 75/100,000 and the prevalence of TB was 97/100,000 in 2012. Extrapulmonary TB represented $4.2 \%(580 / 13,747)$ of all reported TB cases in 2010. 5.8\% (34/580) of extrapulmonary TB occurred in gastrointestinal tract. The incidence of intestinal TB (ITB) was about $0.14 / 100,000$ in 2010. A total of 1,591 IBD patients were registered from 1998 to 2008 in Taiwan (CD: 385). The incidence of CD increased from $0.19 / 100,000$ in 1998 to $0.24 / 100,000$ in 2008 . The prevalence of $\mathrm{CD}$ increased from $0.19 / 100,000$ in 1998 to $1.78 / 100,000$ in $2008 .{ }^{1}$

๑ Copyright 2015. Korean Association for the Study of Intestinal Diseases. All rights reserved.

This is an Open Access article distributed under the terms of the Creative Commons Attribution Non-Commercial License (http://creativecommons.org/licenses/by-nc/3.0)

which permits unrestricted non-commercial use, distribution, and reproduction in any medium, provided the original work is properly cited. 


\section{GASTROENTEROLOGIST'S OPINION}

In geographical regions where ITB and CD coexist, it is difficult to differentiate CD from ITB. The natural course of these two diseases is quite different. ITB is curable when appropriate treatment provided whereas CD is a progressive relapsing illness. Diagnostic approaches rely on clinical, endoscopic, radiological, and pathological findings. Detailed information regarding the symptoms, duration of disease, history of previous $\mathrm{TB}$, extra-intestinal manifestations and chest x-ray findings should be obtained. The tuberculin skin test (TST) had a high false positive rate in a highly BCG vaccinated population as Taiwan. ${ }^{2}$

The colonoscopic features which suggest a diagnosis of CD include anorectal lesions, longitudinal ulcers, aphthous ulcers, cobblestone appearance. Features suggestive of ITB include transverse ulcers, involvement of fewer than four segments, a patulous ileocecal valve and scars or pseudopolyps. ${ }^{3}$ However, the limitations of these scoring systems including small sample size, the positive predictive value was $90 \%$ of cases by scoring system, no validation studies in other geographical population; and most of all, the scoring system would not be useful when there's no colon involved.

\section{RADIOLOGIST'S OPINION}

CT is an essential diagnostic tool as it can be used for the disease localization, evaluating the intestinal and mesenteric extent of the inflammatory process and assessing the extraluminal manifestation. When using the cross sectional images, the bowel wall thickening is usually symmetric and concentric in CD. There will be transmural enhancement

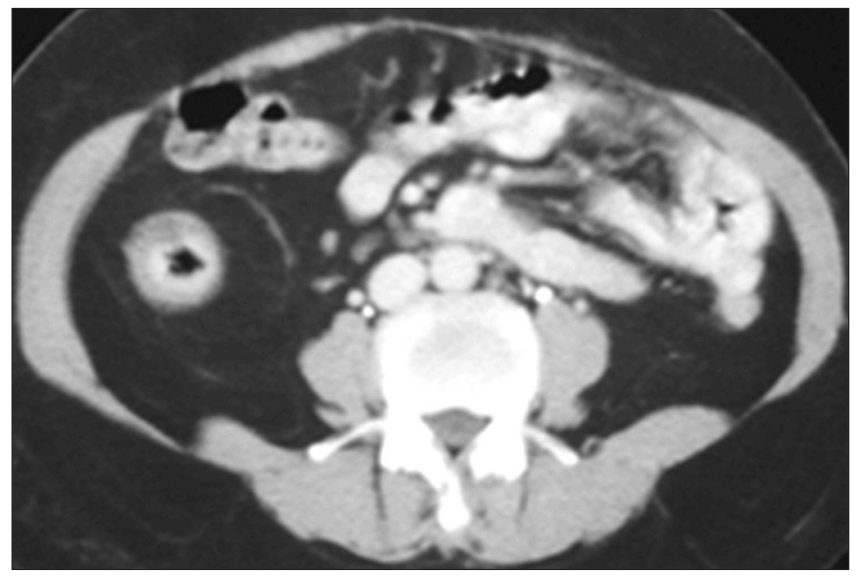

Fig. 1. Abdominal CT finding. Symmetric and concentric wall thickening, with transmural enhancement of terminal ileum of $C D$. in most incidence (Fig. 1). Other features include-intestinal stenosis (Fig. 2), fistula formation (Fig. 3), multiple levels or segmental involvement. It is easy to demonstrate the lessfrequently involved ileocecal region as well as the mural stratification (target sign). ${ }^{4}$ Extramural disease may present as increased mensenteric vascular stranding (comb sign) (Fig. 4) and fibrofatty proliferation in the mesentery; the latter is usually pathognomonic for CD. In ITB, ileocecal region is also a frequent site of involvement. In one study of 130 patients of gastrointestinal $\mathrm{TB}$, the disease located in upper gastrointestinal tract in $8.5 \%$, small bowel in $33.8 \%$ (ileocecal region), large bowel in $22.3 \%$, peritoneum in $30.7 \%$ and liver in $14.6 \%$ were reported. ${ }^{5}$ Ileocecal region involved up to $86 \%$

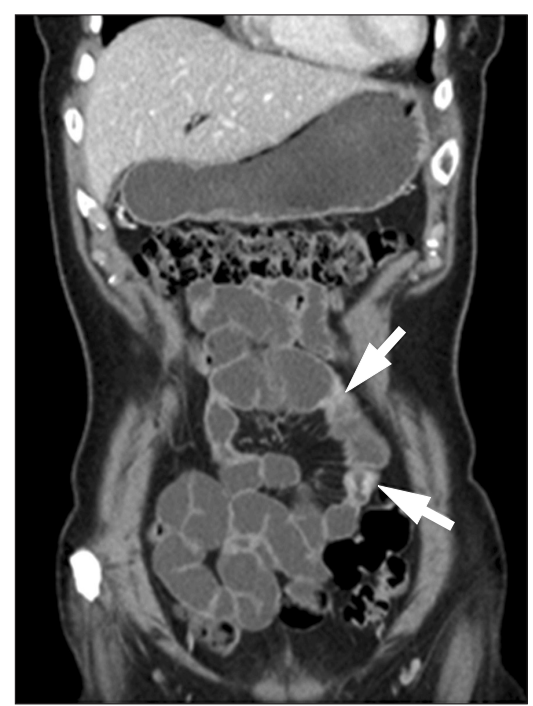

Fig. 2. Abdominal CT finding. Multiple short segment stenoses of small bowel in CD (arrow).

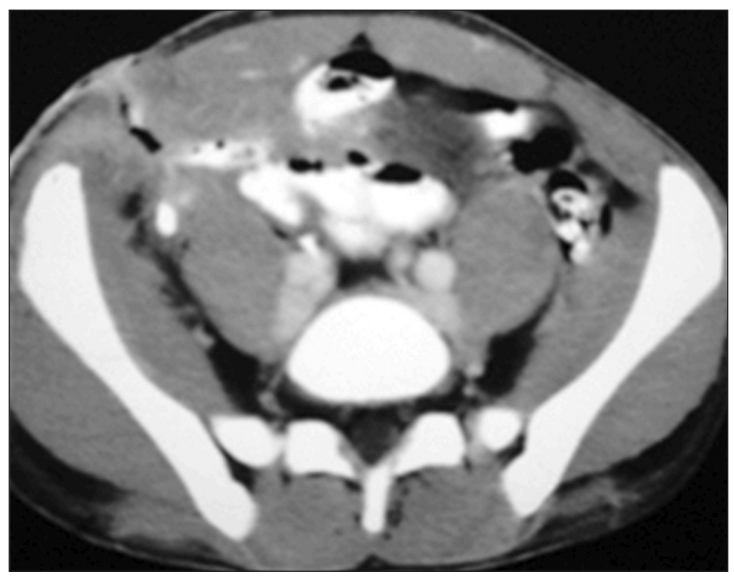

Fig. 3. Abdominal CT finding. Enterocutaneous fistula of CD. 

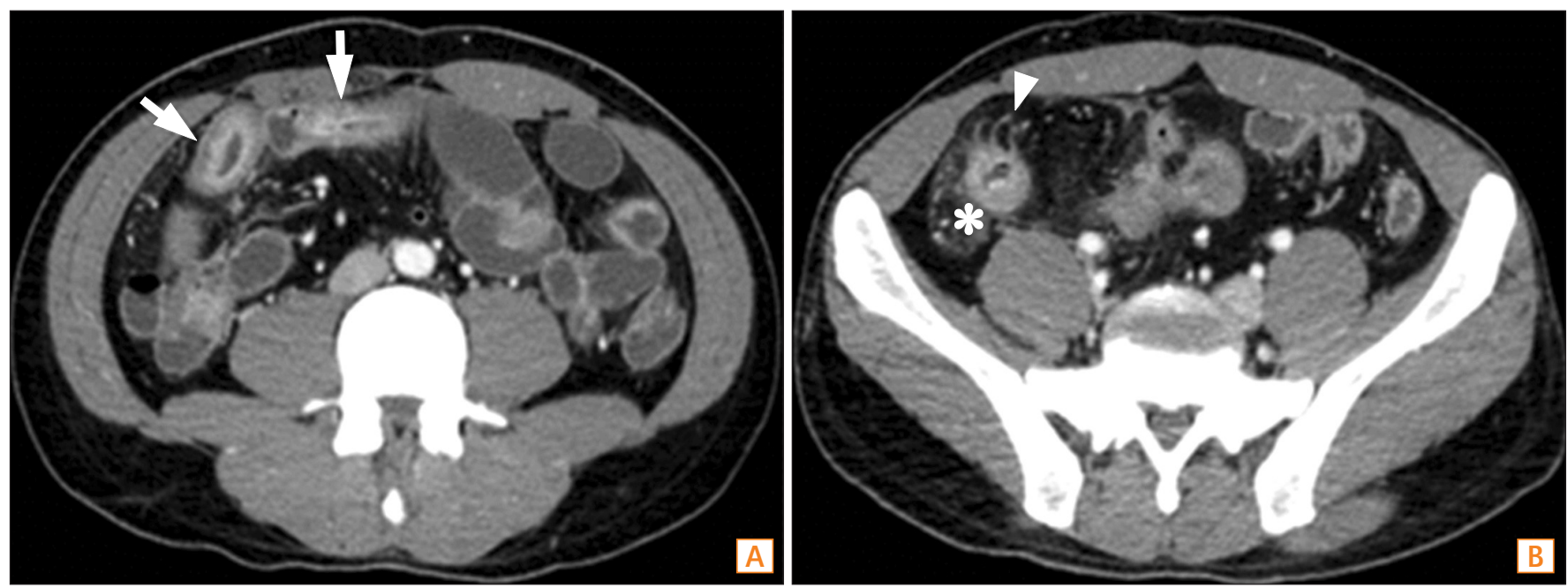

Fig. 4. Abdominal CT findings. (A) The CT image demonstrates bowel wall edema with mural hyperenhancement (arrows). (B) The CT image shows a marked proliferation of fat $(*)$. Extramural disease may present as increased mensenteric vascular stranding (comb sign, arrow head).

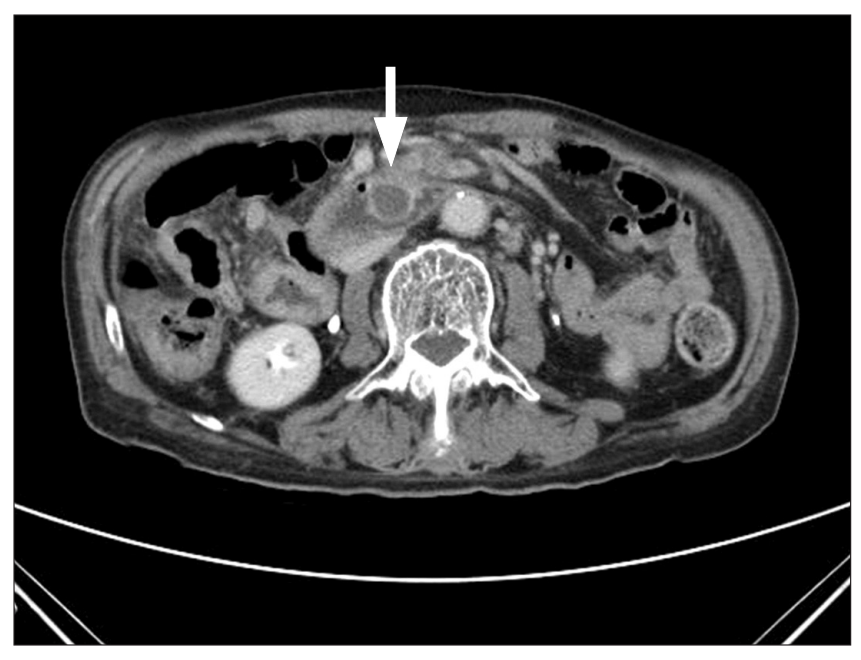

Fig. 5. Abdominal CT finding. Asymmetric thickening of the bowel wall and large necrotic lymph nodes in the mesentery of intestinal tuberculosis.

of ITB patients was also noted. ${ }^{6}$ Asymmetric thickening of the bowel wall and large necrotic lymph nodes (Fig. 5) in the mesentery should raise the suspicion of ITB. ${ }^{7}$

\section{PATHOLOGIST'S OPINION}

To distinguish CD disease from ITB, in addition to the clinical presentation, endoscopic findings, as well as radiological and pathological H\&E staining sections, acid fast stain and/ or TB PCR should be also be studied. According to CD diagnostic criteria in Japan: (1) Major: A) Longitudinal ulcer, B)
Cobblestone-like appearance, C) Noncaseating epithelioid cell granuloma; (2) Minor: a) Irregular-shaped and/or quasicircular ulcers or aphthous ulcerations found extensively in the gastrointestinal tract, b) Characteristic perianal lesions, c) Characteristic gastric and/or duodenal lesions. Definite diagnosis as: (1) Major finding A or B; (2) Major finding C with minor finding a or b; (3) All minor findings $\mathrm{a}, \mathrm{b}$, and $\mathrm{c}^{8}{ }^{8}$

Granuloma is aggregated of histiocytes. The etiology of granuloma included infection and non-infectious reaction. Suppurative granuloma, foreign body granuloma, non-caseating granuloma and caseating granuloma should be differentiated. Histology usually does not allow us to discriminate TB from CD. Clinical differences such as perianal fistulae and types of extraintestinal disease definitely help for further differentiation. A high index of suspicion of TB is required if the patient were immunosuppressed, from high prevalence areas or had history of travel to high prevalence areas. Granulomas were frequently detected in ITB. ${ }^{9}$ In a study compared the pathologic features between $\mathrm{TB}$ and $\mathrm{CD}$, the result showed high frequency of confluent granulomata ( 50 vs. $0 \%$ ), $\geq 10$ granulomata/biopsy site ( 33 vs. $0 \%$ ), caseous necrosis (22 vs. $0 \%$ ) in TB patients. ${ }^{10}$ Absence of focally enhanced colitis and no involvement of sigmoid colon were also independent predictors of ITB. ${ }^{11}$ Higher positivity and reliable results were found in tissue culture, Ziehl-Neelsen stain, and PCR. Ziehl-Neelsen stain and TB PCR could be done by using tissue/DNA from paraffin embedded tissue block. Overall speaking, we should exclude the possibility of TB by any kinds of methods available in our hands. 


\section{INFECTIOUS DISEASE SPECIALIST'S OPINION}

\section{Natural History of TB}

TB is a disease caused by Mycobacterium tuberculosis. Most people infected by $M$. tuberculosis do not develop clinical symptoms, which is called latent TB infection (LTBI). Subjects with LTBI are not infectious. Among them, 2-5\% will develop active disease. The latent period varies from months to decades. Active TB disease can be classified as "open" and "non-open", depending on whether TB bacilli are present in respiratory samples.

\section{Diagnosis of LTBI}

The only method to diagnose LTBI is host immune response. Symptomatology, mycobacteriology and radiology could only exclude active TB disease. The TST is a long-term used screening tool for LTBI. In this test, a mixture of proteins from $M$. tuberculosis were used as antigens. A false-positive result may therefore result from previous BCG vaccination or non-tuberculous mycobacteria infection. Two Interferongamma release assays (IGRAs) are newly developed for the diagnosis of LTBI. The QuantiFERON-TB (QFT) Gold uses ELISA to quantify interferon-gamma (IFN- $\gamma$ ) released from sensitized lymphocytes in whole blood. The other test, the T-SPOT.TB, uses enzyme-linked immunospot to count the number of peripheral blood mononuclear cells that produce IFN- $\gamma$. Both tests use $M$. tuberculosis-specific antigens, including ESAT-6, CFP-10, and TB7.7, and therefore unaffected by BCG vaccination.

Pooled estimates of sensitivity were lowest for the TST (70\%), higher for QFT (76\%), and highest for T-SPOT.TB (88\%). Pooled specificity was $97.7 \%$ for QFT and $92.2 \%$ for TSPOT.TB. Pooled specificity was $66 \%$ for TST. Further analysis showed the specificity of TST was $56 \%$ in BCG-vaccinated population and $98 \%$ in non-BCG vaccinated group. ${ }^{12}$

\section{Risk of Developing Active TB}

Risk factors of TB includes LTBI, advanced human immunodeficiency virus infection, anti-tumor necrosis factor (TNF) agents, old age, healed TB that was not treated, poorly controlled diabetes , tobacco exposure, chronic renal failure, silicosis, gastrectomy, and underweight. ${ }^{13}$

\section{Treatment of ITB and LTBI}

For ITB, therapeutic regimen is the same as pulmonary TB, consisting of Isoniazid (INH; $5 \mathrm{mg} / \mathrm{kg} /$ day), Rifampin (RMP; $10 \mathrm{mg} / \mathrm{kg} /$ day), Ethambutol (EMB; 15-20 mg/kg/day) and Pyrazinamide (PZA; 20-25 mg/kg/day) in the first 2 months (intensive phase), followed by INH and RMP (+EMB) for 4 months (continuation phase).

Four regimens are now recommended for the treatment of LTBI: (1) INH $300 \mathrm{mg}$ (5 mg/kg) daily for 6-9 months; (2) RMP $600 \mathrm{mg}$ (10 mg/kg) daily for 4 months; (3) INH $300 \mathrm{mg}$ (5 mg/kg) plus RMP $600 \mathrm{mg}$ (10 mg/kg) daily for 3 months; and (4) INH $900 \mathrm{mg}$ (15 mg/kg) plus Rifapentine 900 mg (15 $\mathrm{mg} / \mathrm{kg}$ ) weekly for 12 doses. $^{13}$

For IBD patients under biological agents, symptoms and signs of active TB disease should be monitored monthly. Chest radiography and IGRA may be performed every 6 months. Consult TB specialist when necessary.

\section{CONCLUSIONS}

CD and ITB are difficult to differentiate from one another. Combination of clinical manifestations, endoscopic, radiologic and histological features helps us to distinguish these two diseases. TB is one of most communicable diseases in Taiwan. The diagnostic tools of LTBI include TST and IGRA. Screening for LTBI before and regular monitor active TB while using biological agents are highly recommended.

\section{REFERENCES}

1. Wei SC, Lin MH, Tung CC, et al. A nationwide population-based study of the inflammatory bowel diseases between 1998 and 2008 in Taiwan. BMC Gastroenterol 2013;13:166.

2. Fourie PB, Swanevelder JP, Lancaster J. Diagnostic efficiency of the disposable Monotest in detecting tuberculosis infection by setting objective-specific cut-off points for positivity. S Afr Med J 1996;86:151-154.

3. Lee YJ, Yang SK, Byeon JS, et al. Analysis of colonoscopic findings in the differential diagnosis between intestinal tuberculosis and Crohn's disease. Endoscopy 2006;38:592-597.

4. Almadi MA, Ghosh S, Aljebreen AM. Differentiating intestinal tuberculosis from Crohn's disease: a diagnostic challenge. Am J Gastroenterol 2009;104:1003-1012.

5. al Karawi MA, Mohamed AE, Yasawy MI, et al. Protean manifestation of gastrointestinal tuberculosis: report on 130 patients. J Clin Gastroenterol 1995;20:225-232. 
6. Leung VK, Law ST, Lam CW, et al. Intestinal tuberculosis in a regional hospital in Hong Kong: a 10-year experience. Hong Kong Med J 2006;12:264-271.

7. Boudiaf M, Zidi SH, Soyer P, et al. Tuberculous colitis mimicking Crohn's disease: utility of computed tomography in the differentiation. Eur Radiol 1998;8:1221-1223.

8. Hisabe T, Hirai F, Matsui T, Watanabe M. Evaluation of diagnostic criteria for Crohn's disease in Japan. J Gastroenterol 2014;49:93-99.

9. Kim KM, Lee A, Choi KY, Lee KY, Kwak JJ. Intestinal tuberculosis: clinicopathologic analysis and diagnosis by endoscopic biopsy. Am J Gastroenterol 1998;93:606-609.

10. Kirsch R, Pentecost M, Hall Pde M, Epstein DP, Watermeyer G, Friederich PW. Role of colonoscopic biopsy in distinguishing between Crohn's disease and intestinal tuberculosis. J Clin Pathol 2006;59:840-844.
11. Makharia GK, Srivastava S, Das P, et al. Clinical, endoscopic, and histological differentiations between Crohn's disease and intestinal tuberculosis. Am J Gastroenterol 2010;105:642-651.

12. Menzies D, Pai M, Comstock G. Meta-analysis: new tests for the diagnosis of latent tuberculosis infection: areas of uncertainty and recommendations for research. Ann Intern Med 2007;146:340-354.

13. Horsburgh CR, Jr., Rubin EJ. Clinical practice. Latent tuberculosis infection in the United States. N Engl J Med 2011;364:14411448. 